“C 2017 IEEE. Personal use of this material is permitted. Permission from IEEE must be obtained for all other uses, in any current or future media, including reprinting/republishing this material for advertising or promotional purposes, creating new collective works, for resale or redistribution to servers or lists, or reuse of any copyrighted component of this work in other works." 


\section{Performance Analysis of Joint Scheduling in Random Cellular Networks}

\author{
Sinh Cong Lam \\ University of Technology, Sdyney \\ Center of Real Time Information Network \\ Faculty of Engineering and Information Technology \\ Email: SinhCong.Lam@student.uts.edu.au
}

\author{
Kumbesan Sandrasegaran \\ University of Technology, Sdyney \\ Center of Real Time Information Network \\ Faculty of Engineering and Information Technology \\ Email:Kumbesan.Sandrasegaran@uts.edu.au
}

\begin{abstract}
In this paper, we propose a model based on random cellular networks and 3GPP recommendations for LTEAdvanced to analyse the performance of Joint Scheduling in Rayleigh fading channel. The operation of Joint Scheduling is divided into two phases. During the establishment phase, a typical user measures received signals from $K$ coordinated Base Station (BSs) and select the BS with the highest Signalto-Interference-Noise Ratio (SINR). This is followed by the data transmission during the communication phase. The performance of the typical is defined as the conditional probability of coverage probability during the communication phase under condition of the establishment phase. The analytical results which are verified by Monte Carlo simulation can be expanded for both cases of non-Joint Scheduling and the worst case user.
\end{abstract}

Index Terms: Poisson Cellular Network, Coverage Probability, Joint Scheduling

\section{INTRODUCTION}

The rapid increase of Internet-connected mobile subscribers as well as demand for broadband services has driven an urgent need to develop mobile techniques. Coordinated Multipoint (CoMP) transmission and reception [1], [2] have been studied by 3 GPP for LTE-Advanced as a new technique to enhance the quality of the received signals as well as mitigate the interference. The CoMP technique generally can be classified into Joint Transmission and Coordinated Scheduling. Since in Joint Transmission, the typical user receives the date packets from all coordinated BSs at the same time, these packets are always available at these BSs. This significantly leads to an increase in a volume of data traffic on the backhaul link. Meanwhile, every timeslot the centralized scheduling mechanism in Coordinated Scheduling technique determines which BS should transmit data to the typical user. Thus, the data packets are only need to be available at only a BS during a given timeslot, which may reduce the traffic on the backhaul link. Hence, in this paper, we focus on the Coordinated Scheduling.

Figure 1 illustrates an example of coordinated scheduling with two coordinated BSs $(\mathrm{K}=2)$ in which every $K$ BSs are gouped into a group, called a cluster. Conventionally, each cluster using a central scheduling mechanism to control and mitigate the intercell interference within the cluster. The operation of Joint Scheduling with $K=2$ can be detailed as

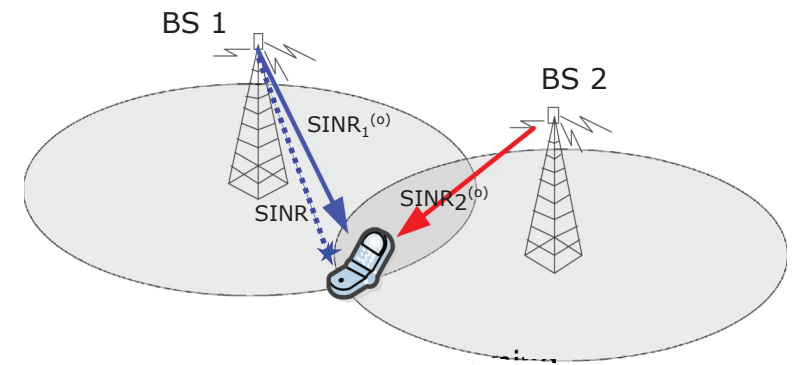

Fig. 1: An example of Coordinated Scheduling with 2 coordinated BSs

follow. First of all, the typical user measures and compares the downlink SINRs on the same RB from two interest BSs, e.g. $S I N R_{1}^{(o)}$ from the BS 1 and $S I N R_{2}^{(o)}$ from the BS 2, which are represented as the solid lines in the figure. The BS with higher SINR, i.e. BS 1 in this case, is selected as the serving BS of the typical user, thus this is reported to the central scheduling mechanism. After that, the data packets are conveyed to the typical user by BS 1 , which is represented as the dashed line.

In the literature, a lot of research work have been conducted to evaluate the performance and optimize Coordinated Scheduling in a hexagonal network layout only, which were sumerized in [3], [4]. Since the deployment of practical cellular networks depends on practical considerations such as radio environment and user distribution, there is an increase in research works based on stochastic geometry models to analyse the network performance [5], [6]. To the best of our knowledge, there is only work on performance analysis of Coordinated Scheduling which was conducted in [7]. In this paper, the worst case user whose distances to three nearst BSs are the same was studied. Hence, there should be more research on on the performance analysis of Cooridnated Scheduling in the PPP network model.

In this paper, we bases on the 3GPP document [1] to propose a model which is used to analyse the performance of Coordinated Scheduling in the PPP networks. Instead of considering one-phase operation as in previous work for the 
hexagonal network layout [8]-[10], we introduce a two-phase operation of Coordinated Scheduling. During the first phase called establishment phase, the typical user measures the received signals from $K$ cooperated BSs and selects the BS with highest SINR as the serving BS. During the second phase called communication phase, the connection between the typical user and the tagged BS is established and data packets are transfered. Hence, the performance of user is defined as a conditional probability of of coverage probability during the communication phase under condition of the establishment phase.

\section{NETWORK MODEL}

We consider the cellular networks using Coordinated Scheduling with a cluster size of $K$ in which the typical user measures and selects one of the $K$ nearest BSs with the highest SINR.

Denote $r_{k}$ is the distance from the typical user to the BS $k$ which is a random variable whose the PDF is given by

$$
f_{R_{k}}\left(r_{k}\right)=\frac{2(\pi \lambda)^{k}}{(k-1) !} r_{k}^{2 k-1} e^{-\pi \lambda r_{k}^{2}}
$$

The joint probability density of $R_{1}, R_{2}, .$. , and $R_{N}$ is defined by $f\left(r_{1}, r_{2}, \ldots, r_{N}\right)$ and given by [12]

$$
f\left(r_{1}, r_{2}, \ldots, r_{N}\right)=(2 \pi \lambda)^{N} e^{-\pi \lambda r_{N}^{2}} \prod_{m=1}^{N} r_{m}
$$

Without loss of generality, it is assumed that $r_{1}<r_{2}<$ $\ldots<r_{K}$.

1) The downlink SINR: The downlink interference at the typical user associated with BS $k$ can be stated as

$$
I_{k}=\underbrace{\sum_{j=1, j \neq k}^{K} P_{j} g_{j} r_{j}^{-\alpha}}_{\text {Intra-Cluster Interference }}+\underbrace{\sum_{j \in \theta^{c}} P_{j} g_{j} r_{j}^{-\alpha}}_{\text {Inter-Cluster Interference }}
$$

in which $g_{j}$ and $r_{j}$ are the power channel gain and distance from the typical user to interfering BS $j$ whose transmit power is $P_{j} ; \alpha$ is the path-loss exponent; $\theta^{c}$ is the set of interfering BSs which belong to adjacent clusters. We denote $\theta$ is the set of BSs in the network, then $\theta=\theta^{c} \bigcup\{1,2, \ldots, K\}$.

Since the BSs in a given cluster fully exchange the channel state information, the Intra-Cell Interference which is originated from the BSs with the same cluster can be controlled by the scheduling mechanism. Meanwhile the Inter-Cluster Interference which is originated from the BSs at adjacent clusters can not be controlled [13]. For simplicity, we assume that the typical user only experiences Inter-Cluster Interference. Thus, the downlink SINR experience the typical user is given by

$$
S I N R_{k}=\frac{P_{k} g_{k} r_{k}^{-\alpha}}{\sum_{j \in \theta^{c}} P_{j} g_{j} r_{j}^{-\alpha}+\sigma^{2}}
$$

in which $\sigma^{2}$ is the power of Gaussian noise.
2) User Association: The typical user connects to the BS $k(1 \leq j \leq K)$ if the BS $k$ provide the highest SINR to the typical user during the establishment phase, i.e $S I N R_{k}^{(o)} \geq$ $S I N R_{j}^{(o)}(\forall 1 \leq j \leq K, j \neq k)$.

With assumption that the typical user measure on the same data channel, all measured SINRs have the same instantaneous interference. Thus, the typical user association problem becomes

$$
g_{k}^{(o)} r_{k}^{-\alpha}>g_{j}^{(o)} r_{j}^{-\alpha} \quad \forall(1 \leq j \leq K, j \neq k)
$$

Proposition 2.1: The average probability in which the user associates with BS $k$ is given by

$$
\begin{aligned}
A_{k}=(2 \pi \lambda)^{K} \int_{0}^{\infty} & \int_{r_{1}}^{\infty} \ldots \int_{r_{K-1}}^{\infty} e^{-\pi \lambda r_{K}^{2}} \prod_{k=1}^{K} r_{k} \\
& \prod_{j=1, j \neq k}^{K} \frac{1}{1+r_{j}^{-\alpha} r_{k}^{\alpha}} d r_{K-1} \ldots d r_{2} d r_{1}
\end{aligned}
$$

Proof: See Appendix A.

\section{Outage Probability}

\section{A. Average Coverage Probability Definition}

The typical user achieves SINR from the BS $k$ during the communication phase as in Equation (4) if it's received signal during the establishment phase is satisfied Equation (5). Hence, the average coverage probability of the typical user associated with BS $k$ at distance $r_{k}$ is defined as the following condition probability

$$
\begin{aligned}
\mathbb{P}_{k}^{(c)}\left(T \mid r_{k}\right) & =\mathbb{P}\left(S I N R_{k}>T \mid \bigcap_{\substack{j=1, j \neq k}}^{K} g_{k}^{(o)} r_{k}^{-\alpha}>g_{j}^{(o)} r_{j}^{-\alpha}\right) \\
& =\frac{\mathbb{P}\left(S I N R_{k}>T, \bigcap_{\substack{j=1 \\
j \neq k}}^{K}, g_{k}^{(o)} r_{k}^{-\alpha}>g_{j}^{(o)} r_{j}^{-\alpha} \mid r_{k}\right)}{\mathbb{P}\left(\bigcap_{\substack{j=1 \\
j \neq k}}^{K} g_{k}^{(o)} r_{k}^{-\alpha}>g_{j}^{(o)} r_{j}^{-\alpha} \mid r_{k}\right)}
\end{aligned}
$$

Since the typical user can associate with any BS, the coverage probability of the typical user at a distance $r_{k}$ from its serving BS $k$ is defined as

$$
\sum_{k=1}^{K} \mathbb{P}\left(\bigcap_{\substack{j=1, j \neq k}}^{K} g_{k}^{(o)} r_{k}^{-\alpha}>g_{j}^{(o)} r_{j}^{-\alpha} \mid r_{k}\right) \mathbb{P}_{k}^{(c)}\left(T \mid r_{k}\right)
$$

Thus, the average coverage probability of the typical user in the network is $\mathcal{P}^{(c)}(T)=$

$$
\sum_{k=1}^{K} \mathbb{E}_{R_{k}}\left[\mathbb{P}\left(S I N R_{k}>T, \bigcap_{\substack{j=1, j \neq k}}^{K} g_{k}^{(o)} r_{k}^{-\alpha}>g_{j}^{(o)} r_{j}^{-\alpha}\right)\right]
$$

in which $f_{R_{k}}\left(r_{k}\right)$ is the PDF of the distance and defined in Equation (1). 
The definition of average coverage probability differentiates from previous work for the hexagonal network layout such as in [13]-[16] since in those work the establishment phase and communication phase were not distinguished.

\section{B. Average Coverage Probability Evaluation}

Theorem 3.1: The average coverage probability of the typical user in the network is given by $P^{(c)}(T)=$

$$
\sum_{k=1}^{K} \underbrace{\mathbb{E}_{R_{j}}}_{1 \leq j \leq K}\left[e^{-\frac{T r_{k}^{\alpha}}{S N R}} e^{-2 \pi \lambda \int_{r_{K}}^{\infty} \frac{T t^{1-\alpha} r_{k}^{\alpha}}{1+T t^{-\alpha} r_{k}^{\alpha}} d t} \prod_{\substack{j=1, j \neq k}}^{K} \frac{1}{1+r_{j}^{-\alpha} r_{k}^{\alpha}}\right]
$$

Proof: See Appendix B.

Employing the joint expectation definition of $K$ random variables whose joint probability density function is defined in Equation 2, the average coverage probability can be re-written as the following equation

$$
\begin{aligned}
P^{(c)}(T)= & (2 \pi \lambda)^{K} \sum_{k=1}^{K} \int_{0}^{\infty} \int_{r_{1}}^{\infty} \ldots \int_{r_{K-1}}^{\infty} e^{-\frac{T r_{k}^{\alpha}}{S N R}} e^{-\pi \lambda r_{K}^{2}} \\
& \prod_{k=1}^{K} r_{k}\left[\prod_{\substack{j=1, j \neq k}}^{K} \frac{e^{-2 \pi \lambda \int_{r_{K}}^{\infty} \frac{T t^{1-\alpha} r_{k}^{\alpha}}{1+T t^{-\alpha} r_{k}^{\alpha}} d t}}{1+r_{j}^{-\alpha} r_{k}^{\alpha}}\right] d r_{K} \ldots . d r_{2} d r_{1}
\end{aligned}
$$

\section{Special Cases}

1) In the case of non-cooperative joint processing: The user connects to the nearest BS $(K=1)$, thus Equation (10) degrades into

$$
\mathcal{P}^{(c)}(T)=\int_{0}^{\infty} 2 \pi \lambda r_{1} e^{-2 \pi \lambda r_{1}^{2}-\frac{T r_{k}^{\alpha}}{S N R}-\pi \lambda r_{k}^{2} \int_{1}^{\infty} \frac{T t^{\alpha / 2}}{1+T t^{\alpha / 2}} d t} d r_{1}
$$

The result of the coverage probability is consistent with the well-known result in [17].

Denote $v(T)=\int_{1}^{\infty} \frac{T t^{\alpha / 2}}{1+T t^{\alpha / 2}} d t$, thus for a given network $v(T)$ is a constant number and can be approximated by using Gauss-Legendre Quadrature and the properties of Gamma function [18].

$$
v(T)=\frac{2}{\alpha} \frac{\pi T^{\frac{2}{\alpha}}}{\sin \left(\frac{2 \pi}{\alpha}\right)}-\sum_{n=1}^{N_{G}} \frac{c_{n}}{2} \frac{T}{\left(\frac{x_{n}+1}{2}\right)^{\frac{2}{\alpha}}+T}
$$

where $N_{G}$ is the degree of the Legendre polynomial, $c_{i}$ and $x_{i}$ are the $i$-th node and weight of the quadrature.

Employing a change of variable $\zeta=\pi \lambda r^{2}$ and the GaussLaguerre Quadrature, we obtain

$$
\mathcal{P}^{(c)}(T) \approx \sum_{i=1}^{N_{G L}} \omega_{i} e^{-t_{i} v(T)}
$$

in which $N_{G L}$ is the degree of the Laguerre polynomial, $t_{i}$ and $w_{i}$ are the $i$-th abscissas and weight of the quadrature.
2) In the case of cooperative joint processing with $K=2$ in the interference-limited network $(\sigma=0)$ with $\alpha=4$ : The average coveage probability of the typical user is obtained by

$$
\begin{aligned}
P^{(c)}(T)=(2 \pi \lambda)^{2} \int_{0}^{\infty} \int_{r_{1}}^{\infty} r_{1} r_{2} e^{-\pi \lambda r_{2}^{2}} & {\left[\frac{e^{-2 \pi \lambda \kappa_{r_{2}}\left(r_{1}\right)}}{1+r_{2}^{-\alpha} r_{1}^{\alpha}}+\frac{e^{-2 \pi \lambda \kappa_{r_{2}}\left(r_{2}\right)}}{1+r_{1}^{-\alpha} r_{2}^{\alpha}}\right] d r_{2} d r_{1} }
\end{aligned}
$$

in which $\kappa_{r_{2}}\left(r_{1}\right)=\int_{r_{2}}^{\infty} \frac{T t^{1-\alpha} r_{1}^{\alpha}}{1+T t^{-\alpha} r_{1}^{\alpha}} d t, \alpha=4$.

By using a change of variable $t=\left(r_{2} / y\right)^{2}$ and reminding that $\alpha=4$, we obtain

$$
\begin{aligned}
\kappa_{r_{2}}\left(r_{1}\right) & =r_{2}^{2} \int_{0}^{1} \frac{T y}{r_{1}^{-4} r_{2}^{4}+T y^{4}} d y \\
& =\frac{\sqrt{T} r_{1}^{2}}{2} \arctan \left(\frac{\sqrt{T} r_{1}^{2}}{r_{2}^{2}}\right)
\end{aligned}
$$

Similarity, we have $\kappa_{r_{2}}\left(r_{2}\right)=\frac{r_{1}^{2}}{2} \sqrt{T} \arctan (\sqrt{T})$.

Considering the first part of the Equation 13 which contains $\kappa_{r_{2}}\left(r_{1}\right)$, the integral can be re-written in the following form

$$
(2 \pi \lambda)^{2} \int_{0}^{\infty} r_{2} e^{-\pi \lambda r_{2}^{2}} \int_{0}^{r_{2}} r_{1} \frac{e^{-\pi \lambda \sqrt{T} r_{1}^{2} \arctan \left(\frac{\sqrt{T} r_{1}^{2}}{r_{2}^{2}}\right)}}{1+r_{2}^{-4} r_{1}^{4}} d r_{1} d r_{2}
$$

This is due to the fact that $\int_{0}^{\infty} \int_{y}^{\infty} f_{X, Y}(x, y) d x d y=$ $\int_{0}^{\infty} \int_{0}^{x} f_{X, Y}(x, y) d y d x, \forall X>Y>0$.

Employing a change of variable $y=r_{1}^{2} / r_{2}^{2}$, the equation above equals

$$
\begin{aligned}
& 2(\pi \lambda)^{2} \int_{0}^{\infty} r_{2} e^{-\pi \lambda r_{2}^{2}} \int_{0}^{1} \frac{e^{-\pi \lambda r_{2}^{2} y \sqrt{T} \arctan (y \sqrt{T})}}{1+y^{2}} d y d r_{2} \\
= & 2(\pi \lambda)^{2} \int_{0}^{1} \frac{1}{1+y^{2}} \int_{0}^{\infty} r_{2}^{3} e^{-\pi \lambda r_{2}^{2}(1+y \sqrt{T} \arctan (y \sqrt{T}))} d r_{2} d y \\
= & \int_{0}^{1} \frac{1}{\left(1+y^{2}\right)(1+y \sqrt{T} \arctan (y \sqrt{T}))} d y
\end{aligned}
$$

Similarity, the second part of Equation 13 which contains $\kappa\left(r_{2}\right) r_{2}$ is obtained by

$$
\int_{0}^{1} \frac{1}{\left(1+y^{2}\right)(1+y \sqrt{T} \arctan (\sqrt{T}))} d y
$$

Consequently, the average coverage probability of the typical user is given by $P^{(c)}(T)=$

$$
\begin{gathered}
\int_{0}^{1} \frac{1}{\left(1+y^{2}\right)}\left[\frac{1}{1+y \sqrt{T} \arctan (\sqrt{T})}\right. \\
\left.+\frac{1}{1+y \sqrt{T} \arctan (\sqrt{T})}\right] d y
\end{gathered}
$$

Interestingly, the average coverage probability of the typical user in this case does not depend on the density of BSs in the network. This is consistent with the previous results which was found in the case of no-coordinated scheduling [17]. 


\section{In the case of worst case user}

In the cellular networks, the typical user which has the same distances to three nearest BSs called the worst case user. The PDF of the distance from the worst case user to it's serving $\mathrm{BS}$ is given by [7]

$$
f_{w}(r)=2(\pi \lambda)^{2} r^{3} e^{-\pi \lambda r^{2}}
$$

Using the results from Equation 9 with $K=3$ and $r_{1}=$ $r_{2}=r_{3}=r$, we obtain the average coverage proability of the worst case user as the following equation

$$
\begin{aligned}
P^{(c)}(T) & =\frac{3}{4} \mathbb{E}_{R}\left[e^{-\frac{T r_{k}^{\alpha}}{S N R}} e^{-2 \pi \lambda \int_{r}^{\infty} \frac{T t^{1-\alpha_{r} \alpha}}{1+T t^{-\alpha_{r} \alpha}} d t}\right] \\
& =\frac{3(\pi \lambda)^{2}}{2} r^{3} e^{-\frac{T r_{k}^{\alpha}}{S N R}} e^{-\pi \lambda r^{2}} e^{-2 \pi \lambda \int_{r}^{\infty} \frac{T t^{1-\alpha_{r} \alpha}}{1+T t^{-\alpha} r^{\alpha}} d t}
\end{aligned}
$$

This result on the worst case user performance differentiates the results conducted in [7] since authors in [7] merged the establishment and communication phases of Joint Scheduling.

\section{Simulation and Discussion}

In this section, we present numerical and simulation results to verify analytical results and the relationship between the coverage threshold and SNR on the network performance.

\section{A. Validation of the Analytical Results}

In oder to validate the analytical results, we compare the average coverage probability of the typical user in Equation 10 with Monte Carlo simulation results. As shown in Fig. 2 , the solid lines representing the analytical results perfectly match with the star points representing the simulation results.

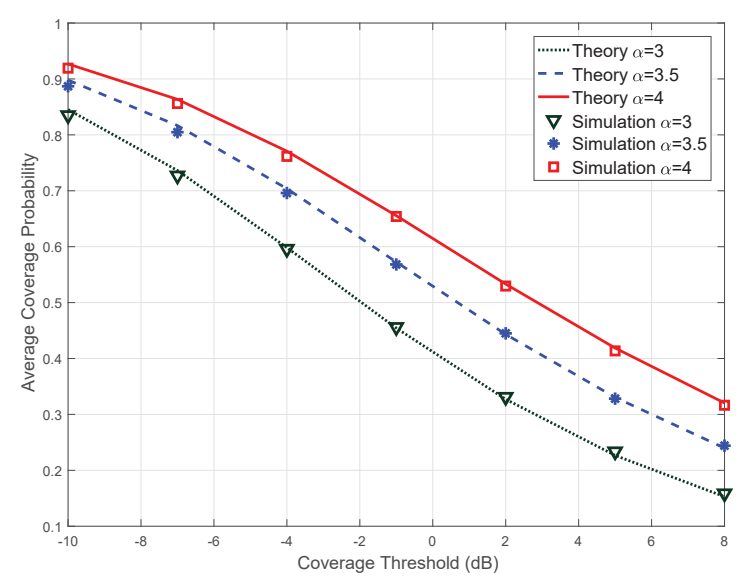

Fig. 2: Average Coverage Probability with different values of $\alpha$ and coverage threshold $(\lambda=0.5$ and $K=2)$

In the Joint Scheduling cellular network systems in which the typical user prefers a connection with the BS in the cluster, the distance from the typical user to the serving BS must be greater than that to the interfering BSs. Therefore, the path loss which is inversely to the distance increases with path loss exponent $\alpha$. Thus, when $\alpha$ increases, the interference signal experiences higher path loss than the serving signal. In other words, the received SINR increases with $\alpha$. Consequently, the average coverage probability of the typical user is proportional to path loss exponent $\alpha$. For example, when $\alpha$ increases from 3 to 4 and coverage threshold $T=2 \mathrm{~dB}$, the average coverage probability increases by approximately $59.9 \%$ from 0.3312 to 0.5296

\section{B. Effects of SNR on the typical user performance}

In this section, the effects of SNR on the typical user performance is visualized as in Figure 3. It is clear that when the performance of the typical user increases with $S N R$. For example, when SNR increases $-10 \mathrm{~dB}$ from to $0.38 \mathrm{~dB}$ and $\lambda=0.5$, the average coverage probability of the typical user increases by $52.52 \%$ from 0.25 to 0.3813 . However, it is observed from the figure that at high values of SNR, e.g. $S N R>20 \mathrm{~dB}$ in the case $\lambda=0.5$, the changes in SNR does not effect on the typical user performance. This is due to the fact that an increase in transmit power of the serving BS also increases in transmit power of the interfering BSs. Hence, at high values of SINR, i.e. transmit power of $\mathrm{BS} \gg$ noise power or $S N R \rightarrow \infty$, the received SINR is approximated by $S I N R_{k} \approx \frac{g_{k} r_{k}^{-\alpha}}{\sum_{j \in \theta^{c}} g_{j} r_{j}^{-\alpha}}$ which does not depend on the transmit power of $\mathrm{BS}$.

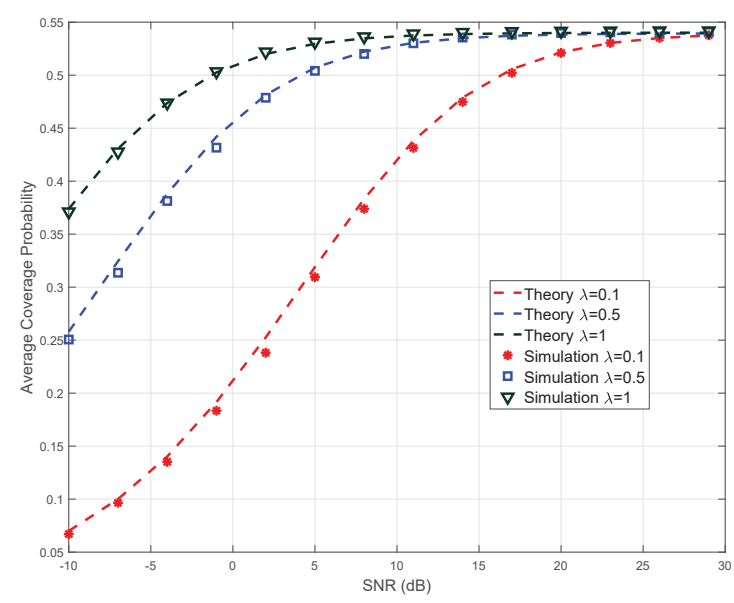

Fig. 3: Average Coverage Probability with different values of SNR and $\lambda$ (Coverage Threshold $T=0 d B$ and $K=2$ )

\section{CONCLUSION}

In this paper, we proposed a two-phase model based PPP to analyse the performance of Joint Scheduling. The proposed model follows the recommendation of 3GPP to separate the operation of Join Scheduling into two phases, called establishment phase and communication phase. Thus, the performance of the typical user is defined as conditional probability of the performance during the communication phase under condition 
of the establishment phase. The analytical method is not only used for the Joint Scheduling but also for non-Joint Scheduling and the worst case user. Furthermore, the paper also states that the performance of the typical user in the network using Joint Scheduling does not depend on the density of BSs which is consistent with the previous works which conducted in the network without Joint Scheduling. To the best of our knowledge, we are the first team working on the performance analysis of the typical user in the PPP networks using Joint Scheduling.

\section{APPENDIX A}

THE AVERAGE PROBABILITY IN WHICH THE TYPICAL USER CONNECTS TO BS $k$

The average probability in which the user associates with $\mathrm{BS} k$ is given by

$$
\begin{aligned}
A_{k} & =\mathbb{P}\left(\bigcap_{j=1, j \neq k}^{K} g_{k}^{(o)} r_{k}^{-\alpha}>g_{j}^{(o)} r_{j}^{-\alpha}\right) \\
& =\mathbb{E}_{G_{j}, R_{j}, R_{k}}\left[\mathbb{P}\left(\bigcap_{j=1, j \neq k}^{K} g_{k}^{(o)}>g_{j}^{(o)} r_{j}^{-\alpha} r_{k}^{\alpha}\right)\right]
\end{aligned}
$$

Since all fading channels are independent Rayleigh random variables,

$$
\begin{aligned}
A_{k} & =\mathbb{E}_{R_{j}, R_{k}, G_{j}}\left[\prod_{j=1, j \neq k}^{K} \mathbb{P}\left(g_{k}^{(o)}>g_{j}^{(o)} r_{j}^{-\alpha} r_{k}^{\alpha}\right)\right] \\
& =\mathbb{E}_{R_{j}, R_{k}}\left[\prod_{j=1, j \neq k}^{K} \mathbb{E}_{G_{j}}\left(e^{-g_{j}^{(o)} r_{j}^{-\alpha} r_{k}^{\alpha}}\right)\right]
\end{aligned}
$$

With assumption that the fading channel has a unit power, e.g. it's probability density function is $\operatorname{PDF}(\gamma)=\exp (-\gamma)$, the it's Moment Generate Function is given by $E\left[e^{-s \gamma}\right]=\frac{1}{1+s \gamma}$. Therefore, the association probability is given by

$$
\begin{aligned}
& A_{k}= \mathbb{E}_{R_{j}, R_{k}}\left[\prod_{j=1, j \neq k}^{K} \frac{1}{1+r_{j}^{-\alpha} r_{k}^{\alpha}}\right] \\
&=(2 \pi \lambda)^{K} \int_{0}^{\infty} \int_{r_{1}}^{\infty} \ldots \int_{r_{K-1}}^{\infty} e^{-\pi \lambda r_{K}^{2}} \prod_{k=1}^{K} r_{k} \\
& \prod_{j=1, j \neq k}^{K} \frac{1}{1+r_{j}^{-\alpha} r_{k}^{\alpha}} d r_{K-1} \ldots d r_{2} d r_{1}
\end{aligned}
$$

The Proposition is proved.

\section{APPENDIX B}

THE COVERAGE PROBABILITY OF THE TYPICAL USER ASSOCIATED WITH BS $k$

The conditional probability in Equation (8) can be evaluated by using the following steps

$$
\mathbb{P}\left(g_{k}>T \frac{\sum_{j \in \theta^{c}} P_{j} g_{j} r_{j}^{-\alpha}+\sigma^{2}}{P_{k} r_{k}^{-\alpha}}, \bigcap_{\substack{j=1, j \neq k}}^{K} g_{k}^{(o)}>g_{j}^{(o)} r_{j}^{-\alpha} r_{k}^{\alpha}\right)
$$

Since the channel power gains are independent exponential random variables,

$$
\begin{aligned}
& e^{-\frac{T r_{k}^{\alpha}}{S N R}} \mathbb{E}\left[e^{-T \sum_{j \in \theta^{c}} g_{j} r_{j}^{-\alpha} r_{k}^{\alpha}-\sum_{\substack{j=1 \\
j \neq k}}^{K} g_{j}^{(o)} r_{j}^{-\alpha} r_{k}^{\alpha}}\right] \\
& =\stackrel{(\mathrm{a})}{=} \mathbb{E}_{R_{j}}\left[\prod_{j \in \theta^{c}} \mathbb{E}_{G_{j}}\left[e^{-T r_{j}^{-\alpha} r_{k}^{\alpha} g_{j}}\right] \prod_{\substack{j=1, j \neq k}}^{K} \mathbb{E}_{G_{j}^{(o)}}\left[e^{-g_{j}^{(o)} r_{j}^{-\alpha} r_{k}^{\alpha}}\right]\right] \\
& \stackrel{\text { (b) }}{=} \underbrace{\mathbb{E}_{R_{j}}}_{\substack{1 \leq j \leq K \\
j \neq k}}[\underbrace{\mathbb{E}_{R_{j}}}_{j>K}\left[\prod_{j \in \theta^{c}} \frac{1}{1+T r_{j}^{-\alpha} r_{k}^{\alpha}}\right] \prod_{\substack{j=1, j \neq k}}^{K} \frac{1}{1+r_{j}^{-\alpha} r_{k}^{\alpha}}] \\
& \stackrel{\text { (c) }}{=} \underbrace{\mathbb{E}_{R_{j}}}_{\substack{1 \leq j \leq K \\
j \neq k}}\left[e^{-2 \pi \lambda \int_{r_{K}}^{\infty} \frac{T t^{1-\alpha} r_{k}^{\alpha}}{1+T t^{-\alpha} r_{k}^{\alpha}} d t} \prod_{\substack{j=1, j \neq k}}^{K} \frac{1}{1+r_{j}^{-\alpha} r_{k}^{\alpha}}\right]
\end{aligned}
$$

in which $(a)$ follows the assumption that all fading channels are random variables; $(b)$ due to the assumption that $g_{j}$ is an exponential random variable; $(c)$ obtains by using the properties of Probability Generating Function.

Substituting 19 into 8 , the Theorem is proved.

\section{REFERENCES}

[1] 3GPP TR 36.819 V11.1.0, “Coordinated multi-point operation for LTE physical layer aspects ," December 2011.

[2] L. Daewon, S. Hanbyul, B. Clerckx, E. Hardouin, D. Mazzarese, S. Nagata, and K. Sayana, "Coordinated multipoint transmission and reception in lte-advanced: deployment scenarios and operational challenges," IEEE Commun. Mag., vol. 50, no. 2, pp. 148-155, 2012.

[3] G. Y. Li, N. Jinping, L. Daewon, F. Jiancun, and F. Yusun, "Multi-Cell Coordinated Scheduling and MIMO in LTE," IEEE Commun. Surveys \& Tutorials, vol. 16, no. 2, pp. 761-775, 2014.

[4] S. Bassoy, H. Farooq, M. A. Imran, and A. Imran, "Coordinated multipoint clustering schemes: A survey," IEEE Communications Surveys Tutorials, vol. PP, no. 99, pp. 1-1, 2017.

[5] H. ElSawy, E. Hossain, and M. Haenggi, "Stochastic Geometry for Modeling, Analysis, and Design of Multi-Tier and Cognitive Cellular Wireless Networks: A Survey," IEEE Commun. Surveys Tutorials, vol. 15 , no. 3, pp. 996-1019, Third 2013.

[6] M. Haenggi, Stochastic Geometry for Wireless Networks . Cambridge Univ. Press, November 2012.

[7] S. Y. Jung, H. k. Lee, and S. L. Kim, "Worst-Case User Analysis in Poisson Voronoi Cells," IEEE Commun. Lett., vol. 17, no. 8, pp. 15801583, August 2013.

[8] L. Venturino, N. Prasad, and X. Wang, "Coordinated scheduling and power allocation in downlink multicell ofdma networks," IEEE Transactions on Vehicular Technology, vol. 58, no. 6, pp. 2835-2848, July 2009.

[9] K. Shen and W. Yu, "A coordinated uplink scheduling and power control algorithm for multicell networks," in 2015 49th Asilomar Conference on Signals, Systems and Computers, Nov 2015, pp. 1305-1309.

[10] Y. Wang, X. Su, D. Choi, and C. Choi, "Coordinated scheduling algorithm for system utility maximization with heterogeneous qos requirements in wireless relay networks," IEEE Access, vol. 4, pp. 83518361, 2016.

[11] G. Nigam, P. Minero, and M. Haenggi, "Coordinated Multipoint Joint Transmission in Heterogeneous Networks," IEEE Transactions on Communications, vol. 62, no. 11, pp. 4134-4146, Nov 2014.

[12] D. Moltchanov, "Survey paper: Distance distributions in random networks," Ad Hoc Netw., vol. 10, no. 6, pp. 1146-1166, Aug. 2012 [Online]. Available: http://dx.doi.org/10.1016/j.adhoc.2012.02.005 
[13] P. Marsch and G. P. Fettweis, Coordinated Multi-Point in Mobile Communications: From Theory to Practice, 1st ed. New York, NY, USA: Cambridge University Press, 2011.

[14] X. Zhang and M. Haenggi, "A stochastic geometry analysis of inter-cell interference coordination and intra-cell diversity," IEEE Transactions on Wireless Communications, vol. 13, no. 12, pp. 6655-6669, Dec 2014.

[15] C. Li, J. Zhang, M. Haenggi, and K. B. Letaief, "User-centric intercel interference nulling for downlink small cell networks," IEEE Transac tions on Communications, vol. 63, no. 4, pp. 1419-1431, April 2015.

[16] S. Govindasamy and I. Bergel, "Uplink performance of multi-antenna cellular networks with co-operative base stations and user-centric clustering," April 2017.

[17] J. G. Andrews, F. Baccelli, and R. K. Ganti, "A new tractable model for cellular coverage," in 2010 48th Annu. Allerton Conference on Communication, Control, and Computing (Allerton), pp. 1204-1211.

[18] S. C. Lam, R. Heidary, and K. Sandrasegaran, "A closed-form expression for coverage probability of random cellular network in composite Rayleigh-Lognormal fading channels," in 2015 Proc. IEEE Int. Telecommun. Networks and Applicat. Conf.(ITNAC), 2015, pp. 161-165. 